\title{
Heterogeneous sources model for DSL
}

\section{access multiplexers}

B. Steyaert, J. Walraevens, D. Fiems, D. De Vleeschauwer and H. Bruneel

Abstract: We study a Digital Subscriber Line Access Multiplexer (DSLAM) that multiplexes streaming Packetized Telephony (PT) and/or Video-on-Demand (VoD) traffic. To this end, the distribution of the amount of work in a discrete-time single-server queueing model with heterogeneous sources and general packet lengths is calculated. Obtaining actual data concerning the distribution of this quantity is notoriously hard. We outline how the tail distribution can be extracted from the steady-state probability generating function (pgf) through a multiple-pole series expansion.

Introduction: We study the behavior of a DSLAM [1] supporting a large number of subscribers with streaming audio and/or video applications, where different PT and VoD devices can have different codec bit rates. To this end, we first analyze a discrete-time queueing model where (each application of) each subscriber is modeled as a traffic source that generates packets with generally distibuted lengths and according to a 2-state discrete-time Markov modulated Bernoulli process (MMBP) [2]. The analysis of such a system often is a difficult task, due to the fact that the sources are heterogeneous (PT and VoD sources with different codec rates for instance generate packets at a different rate and/or of different length), and because of the 
time-correlated behaviour that each of the individual sources exhibits. We adopt a generatingfunction-based solution method (the details of which fall beyond the scope of this letter, for more details we refer to [3]) and derive the tail distribution of the amount of work in the buffer. Emphasis is put on the extraction of actual numerical data from the pgf since the number of sources (i.e., the state space) is usually high, an issue of practical interest that is all too often neglected in the literature. Additionally, we show that the standard dominant single-pole approximation for the inversion of the pgf is far from accurate and therefore propose a multiplepole extension.

Queueing model: Consider a discrete-time infinite-capacity single-server queue fed by $N$ sources that can switch between 2 states on slot boundaries. Identical sources are grouped into $K$ classes according to their traffic characteristics, and we let $N_{k}$ represent the number of sources belonging to class $k$. A source of class $k$ and its transition parameters are shown in Fig. 1 $\left(p_{i 1, k}=1-p_{i 2, k}, i=1,2\right)$. When in the $\mathrm{ON}$ state during a slot, the source generates a packet with probability $\gamma_{k}$, while the source is inactive when in the OFF state. The user activity $\sigma_{k}$, being the fraction of time that a class- $k$ subscriber is in the ON state, equals $p_{21, k} /\left(p_{21, k}+p_{12, k}\right)$. An important quantity in our source description is the $2 \times 2$ matrix $\mathbf{Q}_{\mathbf{k}}(z)$, defined as

$$
\mathbf{Q}_{\mathbf{k}}(z)=\left[\begin{array}{cc}
\left(1-\gamma_{k}+\gamma_{k} z\right) p_{11, k} & p_{12, k} \\
\left(1-\gamma_{k}+\gamma_{k} z\right) p_{21, k} & p_{22, k}
\end{array}\right] \text {. }
$$

with eigenvalues $\lambda_{j, k}(z), 1 \leq j \leq 2$. We further define $S_{k}(z)$ as the pgf of the class- $k$ packet lengths (expressed in slots). If class- $k$ packets have a constant length equal to $L_{s, k}$ slots, as is often assumed (for the DSLAM), $S_{k}(z)=z^{L_{s, k}}$.

[Fig. 1 about here.]

The analysis of this queue can be carried out by resorting to a pgf-approach [3]. Assuming that the equilibrium condition $\rho \triangleq \sum_{k=1}^{K} N_{k} \sigma_{k} \gamma_{k} S_{k}^{\prime}(1)<1$ is satisfied, this leads to the following 
expression for the steady-state pgf $U(z)$ of the work in the buffer at slot boundaries $(\mathbf{m}=$ $\left.\left(m_{1} \ldots m_{K}\right)\right)$

$$
U(z)=\sum_{m_{1}=0}^{N_{1}} \cdots \sum_{m_{K}=0}^{N_{K}} \frac{(1-\rho)(z-1)}{z-\prod_{k=1}^{K}\left(\lambda_{1, k}\left(S_{k}(z)\right)^{m_{k}} \lambda_{2, k}\left(S_{k}(z)\right)^{N_{k}-m_{k}}\right)} \varepsilon_{\mathbf{m}}(z) .
$$

The functions $\varepsilon_{\mathbf{m}}(z)$ contain boundary probabilities that can be calculated by solving a set of linear equations. The size of this set of equations can become extremely high, and therefore we instead use an explicit approximation in terms of the eigenvalues $\lambda_{j, k}(z)$ and the corresponding eigenvectors that has been shown to be sufficiently accurate [3]. The tail distribution of the queue length is calculated from the pgf (2) by invoking a dominant-pole approximation. However, the single-pole approximation that is often adopted in the literature is not a sufficiently accurate approximation here. Hence we propose a multiple-pole extension leading to the following formula for the tail probabilities:

$$
\operatorname{Pr}[u>Q] \cong \sum_{m_{1} \leq N_{1}} \cdots \sum_{m_{K} \leq N_{K}} \frac{-C_{\mathbf{m}}}{z_{\mathbf{m}}-1} z_{\mathbf{m}}^{-Q-1}
$$

where the pole $z_{\mathbf{m}}$ of $U(z)$ is the real solution of

$$
z=\prod_{k=1}^{K}\left(\lambda_{1, k}\left(S_{k}(z)\right)^{m_{k}} \lambda_{2, k}\left(S_{k}(z)\right)^{N_{k}-m_{k}}\right) \quad, \quad z_{\mathbf{m}}>1
$$

and $C_{\mathbf{m}}$ its corresponding residue, which is readily calculated from (2). The term for $\mathbf{m}=$ $\left(N_{1} \cdots N_{K}\right)$ in (3) corresponds to the single-pole approximation, while decreasing values of $\mathbf{m}$ correspond to 'less dominant' poles. For any given value of $Q$, subsequent terms in (3) should be calculated, until a sufficient accuracy is achieved according to an appropriate stopping criterion.

DSLAM buffer: We assume a DSLAM. Usually part of the bandwidth is reserved for PT traffic, while the rest can be used by the VoD sources. Therefore, we can assume that both types of traffic do not interact. We therefore focus here on PT sources only. Note that a similar treatment is possible for VoD traffic or in case the traffic of both types interact. In a first example 
we show the necessity for the multiple-pole approximation. Consider $N=700$ homogeneous PT sources that are multiplexed on an $R=10 \mathrm{Mb} / \mathrm{s}$ output link. The input bit rate of an active source is $80 \mathrm{~kb} / \mathrm{s}$ and the mean length of an active period equals 120 seconds. Packets have a length of 200 bytes and the subscriber activity $\sigma$ is 0.1 . These parameters are easily mapped to the parameters of the queueing model in the previous section. Hereby, slot lengths are equal to the transmission time of 100 bytes. Fig. 2 compares the single- and multiple-pole approximations for the work complementary cumulative distribution (CCD) obtained from (3). This figure shows that the single-pole approximation, that is all too often adopted in the literature in the performance assessment of this type of systems, is a too coarse an approximation for the queue length CCD to be of value, and that taking into account multiple poles is a necessity.

[Fig. 2 about here.]

In a second figure 3, we assume two types of PT sources, one with a bitrate of $64 \mathrm{~kb} / \mathrm{s}$ and packet lengths of 180 bytes and the other with a bitrate of $16 \mathrm{~kb} / \mathrm{s}$ and 60 -byte packets $(\alpha=0.1$ for both types). We assume that the loads of both types are restricted by the requirement that $\operatorname{Pr}[u>30 m s]<10^{-4}$, which is a usual constraint to ensure interactive telephone calls of acceptable quality. We have drawn 4 curves of admissible loads for 4 different output link rates. From this figure it is seen that the multiplexing gain of small bitrate codecs is larger, and that only acceptable multiplexing gains are achieved when the output link rate is at least a couple of $\mathrm{Mb} / \mathrm{s}$. This type of results is very useful for an access network operator to perform admission control. We note that these figures can be generated almost instantly, while the exact solution would take a lot more time. For instance for the middle data point of the curve with an output link rate equal to $8.192 \mathrm{Mb} / \mathrm{s}$, the arrival model contains 488607 states, and the exact queueing analysis becomes infeasible.

[Fig. 3 about here.] 
Conclusion: We have focused on a discrete-time single-server queueing model with 2-state heterogeneous sources and general packet lengths, to study a DSLAM buffer with a large number of subscribers generating PT and VoD streaming traffic. An effective method for calculating the tail distribution of the work in the buffer from its generating function was demonstrated, by introducing appropriate approximations for the boundary probabilities and by adopting a multiple-pole series expansion. This approach avoids to a large extent the problem of having to deal with a large state space. 


\section{References}

1 Van Hoey, G., De Vleeschauwer, D., Steyaert, B., Inghelbrecht, V. and Bruneel, H.: 'Benefit of Admission Control in Aggregation Network Dimensioning for Video Services', Lecture Notes in Computer Science, 2004, 3042, pp. 357-368

2 IZqUieRdo, M.R. and REEVES, D.S.: 'A survey of statistical source models for variable-bit-rate compressed video', Multimedia Systems, 1999, 7, pp. 199-213

3 Steyaert, B.: 'Analysis of Generic Discrete-Time Buffer Models with Irregular Packet Arrival Patterns' PhDthesis, Ghent University, 2008

\section{Author's affiliations}

B. Steyaert, J. Walraevens, D. Fiems, D. De Vleeschauwer and H. Bruneel (Ghent University, Department of Telecommunications and Information Processing, SMACS Research Group, St.-Pietersnieuwstraat 41,B-9000 Gent, Belgium). D. De Vleeschauwer (Alcatel-Lucent Bell, Copernicuslaan 50, 2018 Antwerp, Belgium). This work has been carried out in the framwork of the Q-MATCH project sponsored by the Flemish Institute for the Promotion of Scientific and Technological Research in the Industry (IWT). The second and third author are Postdoctoral Fellows with the Research Foundation Flanders (FWO-Vlaanderen), Belgium.

\section{E-mail}

bs@telin.ugent.be 


\section{List of Figures}

1 The ON/OFF class- $k$ source model $\ldots \ldots \ldots$

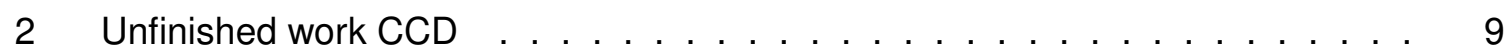

3 Admissible loads of two types of PT sources . . . . . . . . . . . . . . . . 10 


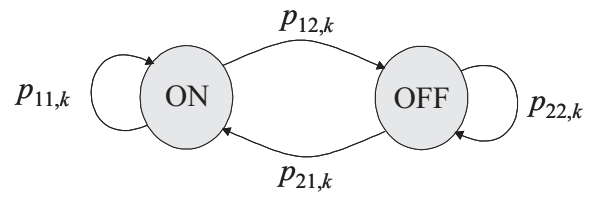

Fig. 1 The ON/OFF class- $k$ source model 


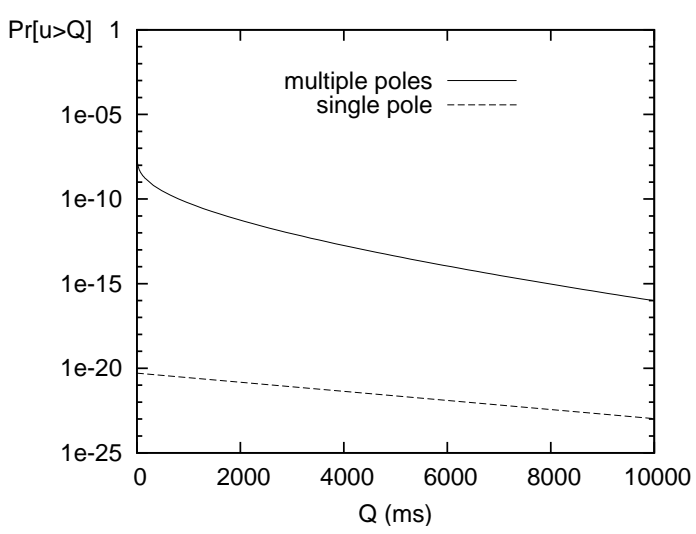

Fig. 2 Unfinished work CCD 


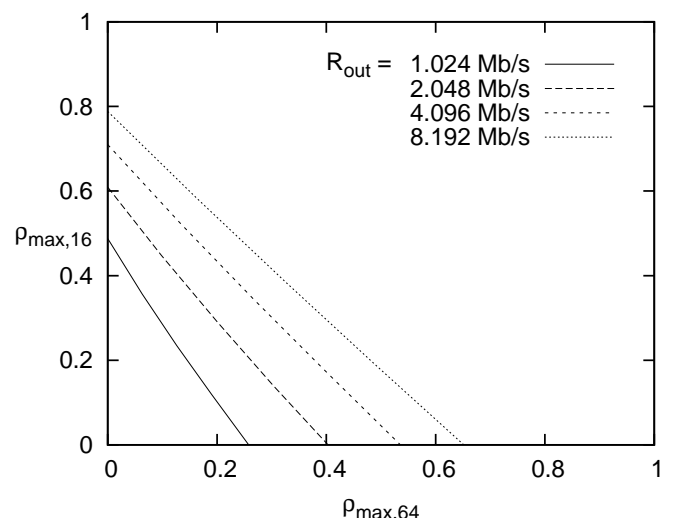

Fig. 3 Admissible loads of two types of PT sources 